Acta Math. Hungar., 125 (4) (2009), 299-326.

DOI: $10.1007 / \mathrm{s} 10474-009-9023-\mathrm{z}$

First published online July 6, 2009

\title{
ON GROWTH OF NORMS OF NEWTON INTERPOLATING OPERATORS
}

\author{
A. P. GONCHAROV
}

Department of Mathematics, Bilkent University, 06800 Ankara, Turkey e-mail: goncha@fen.bilkent.edu.tr

(Received January 22, 2009; revised March 12, 2009; accepted March 13, 2009)

\begin{abstract}
We consider the problem of growth of the sequence of Lebesgue constants corresponding to the Newton interpolation and estimate the growth of this sequence in the case of a nested family of Chebyshev's points.
\end{abstract}

\section{Introduction}

Let $X=\left(x_{k}^{(n)}\right)_{n=1, k=1}^{\infty}{ }^{n}$ be an infinite triangular array of nodes in $[-1,1]$. Let $\Lambda_{N}(X)$ denote the $N$-th Lebesgue constant, that is the uniform norm of the Lagrange interpolating operator defined by the points $\left(x_{k}^{(N+1)}\right)_{k=1}^{N+1}$. It is well-known that the sequence $\left(\Lambda_{N}(X)\right)_{N=0}^{\infty}$ has at least logarithmic growth and that the Chebyshev array $T$ is close to the optimal choice. Now suppose that the array $X$ is nested, that is, any row of $X$ consists of the previous row plus one more value. What is the growth of $\left(\Lambda_{N}(X)\right)_{N=0}^{\infty}$ in this case?

Starting from the classical papers by C. Runge, S. Bernstein, and G. Faber, the problem of approximating properties of Lagrange interpolation has attracted attention of many mathematicians. A variety of results

Key words and phrases: Lebesgue constant, Newton interpolation.

2000 Mathematics Subject Classification: primary 41A05; secondary 41A44. 
concerning asymptotic behavior of the Lebesgue constant were obtained for different arrays (see e.g. [1], [4], [7]), as well as for different metrics. It is rather surprising that the naturally arising corresponding problem for the Newton interpolation was out of consideration. In this respect we can mention only Problem II in [3], where the question of existence of a nested interpolation process for a fixed function $f \in C[0,1]$ was posed.

How to choose a sequence $X=\left(x_{n}\right)_{n=1}^{\infty} \subset[-1,1]$ with a moderate growth of $\left(\Lambda_{N}(X)\right)_{N=0}^{\infty}$ ? One can suppose that such a sequence must at least approximate the equilibrium arcsine distribution $d \mu_{e}(x)=\frac{d x}{\pi \sqrt{1-x^{2}}}$. This means that the finite measures $\frac{1}{n} \sum_{k=1}^{n} \delta_{x_{k}}$, where $\delta_{x_{k}}$ stands for the unit mass located at $x_{k}$, converge to $d \mu_{e}$ in the weak ${ }^{*}$ topology of measures. For example, the Leja sequence $L$ has this approximating property (see e.g. [6], T.V.1.1). R. Taylor and V. Totik [8] proved that the sequence $\left(\Lambda_{N}(L)\right)_{N=0}^{\infty}$ has subexponential growth, that is $\frac{\log \Lambda_{N}(L)}{N} \rightarrow 0$, as $N \rightarrow \infty$. Here and subsequently, log denotes the natural logarithm.

The Leja sequence is a nested analog of the array $F$ of Fekete points (see e.g. [6] for the definition of Fekete points). Even for the set $[-1,1]$, the exact position of the Leja points is not known, whereas the corresponding $n$ th Fekete points are the zeros of the Jacobi polynomial $P_{n-2}^{(1,1)}$ together with the points \pm 1 (see e.g. [7], T.6.7.1). It should be noted that asymptotically $\Lambda_{N}(F) \geqq N^{3 / 2}$ (see e.g. [4], Ch. 10, Corollary 13). On the other hand, for the Chebyshev array $T$ we have $\Lambda_{N}(T) \leqq \frac{2}{\pi} \log N+1$ (see e.g. [5], Theorem 1.2). Thus, a nested analog of the array $T$ can be considered as a possible choice for the desired sequence $X$.

Here we arrange in a special manner the zeros of the Chebyshev polynomials $\left(T_{3^{s}}\right)_{s=0}^{\infty}$, and show that

$$
\Lambda_{N}(X)=O\left(\exp \left(\frac{\log ^{2} N}{\log 3}+8 \log N\right)\right)
$$

for such nodes. The constant involved in this estimate is not exact, but for a subsequence $\left(N_{s}\right)$ we have the lower bound $\Lambda_{N_{s}}(X) \geqq \exp \left(\frac{\log ^{2} N_{s}}{\log 27}-5 \log N_{s}\right)$.

\section{The choice of a sequence of interpolating nodes}

The Chebyshev polynomials $T_{N}(x)=\cos (N \arccos x)$ have the property $T_{N}\left(T_{M}(x)\right)=T_{N M}(x)$. If $M$ and $N$ are odd, then the polynomial $T_{N M}$ preserves all zeros of $T_{M}$. Therefore one can arrange a nested family of zeros of a subsequence of the Chebyshev polynomials. Here we consider the polyno- 
mials $T_{3^{s}}$ for $s \in \mathbb{N}_{0}:=\{0,1,2, \ldots\}$. Let $\theta_{j}^{(s)}=\frac{2 j-1}{3^{s}} \frac{\pi}{2}$ for $j=1,2, \ldots, 3^{s}$. Then $\left\{\cos \theta_{j}^{(s)}\right\}_{j=1}^{3^{s}}$ is the set of zeros of $T_{3^{s}}$. Let $Z_{0}=\{0\}$ and for $s$ $\in \mathbb{N}$ let $Z_{s}$ denote the zeros of $T_{3^{s}}$ that are not zeros of $T_{3^{s-1}}$. That is $Z_{s}=\left\{z_{j}=\cos \theta_{j}^{(s)}: j=1,3,4,6,7, \ldots, 3^{s}-3,3^{s}-2,3^{s}\right\}$. Then $Z_{s}$ can be represented as the union of the set $Z_{s}^{-}=\left\{\cos \left(\theta_{j}^{(s-1)}+\frac{\pi}{3^{s}}\right)\right\}_{j=1}^{3^{s-1}}$ with the set $Z_{s}^{+}=\left\{\cos \left(\theta_{j}^{(s-1)}-\frac{\pi}{3^{s}}\right)\right\}_{j=1}^{3^{s-1}}$, where the superscripts \pm are related to the position of the point from $Z_{s}$ with respect to the corresponding value from $\cup_{k=0}^{s-1} Z_{k}$.

We enumerate all points from $X:=\cup_{s=0}^{\infty} Z_{s}$ in the following way: let $x_{1}=0, x_{2}=\cos \frac{5 \pi}{6}, x_{3}=\cos \frac{\pi}{6}$ and if the points $\left(x_{k}\right)_{k=1}^{3^{s}}$ with $x_{k}=\cos \psi_{k}$ are chosen, then let $x_{3^{s}+k}=\cos \left(\psi_{k}+\pi \cdot 3^{-s-1}\right), x_{2 \cdot 3^{s}+k}=\cos \left(\psi_{k}-\pi \cdot 3^{-s-1}\right)$ for $k=1,2, \ldots, 3^{s}$. Thus, we include at first all points from the set $Z_{s}^{-}$in the order given by $\cup_{k=0}^{s-1} Z_{k}$ in the sequence $\left(x_{k}\right)_{k=1}^{\infty}$ and afterwards we do so for the corresponding points from $Z_{s}^{+}$. For the convenience of the reader, here we give some values of $\psi_{k}$ :

$$
\begin{gathered}
\psi_{1}=\frac{\pi}{2}, \quad \psi_{2}=\frac{\pi}{2}+\frac{\pi}{3}, \quad \psi_{3}=\frac{\pi}{2}-\frac{\pi}{3}, \quad \psi_{4}=\frac{\pi}{2}+\frac{\pi}{9}, \quad \psi_{5}=\frac{\pi}{2}+\frac{\pi}{3}+\frac{\pi}{9} \\
\psi_{6}=\frac{\pi}{2}-\frac{\pi}{3}+\frac{\pi}{9}, \quad \psi_{7}=\frac{\pi}{2}-\frac{\pi}{9}, \quad \psi_{10}=\frac{\pi}{2}+\frac{\pi}{27} \\
\psi_{100}=\psi_{3^{4}+19}=\psi_{2 \cdot 3^{2}+1}+\frac{\pi}{3^{5}}=\frac{\pi}{2}-\frac{\pi}{3^{3}}+\frac{\pi}{3^{5}}
\end{gathered}
$$

Let us represent the angle $\psi_{n}$ as a combination of $\delta_{k}=\pi 3^{-k}$ with $k \in \mathbb{N}$. By construction of the sequence $\left(\psi_{n}\right)_{n=1}^{\infty}$, we see that $\psi_{2}=\psi_{1}+\delta_{1}$, $\psi_{3}=\psi_{1}-\delta_{1}$ and for $s \in \mathbb{N}$ we have $\psi_{3^{s}+k}=\psi_{k}+\delta_{s+1}, \psi_{2 \cdot 3^{s}+k}=\psi_{k}-\delta_{s+1}$ for $k=1,2, \ldots, 3^{s}$. For a general formula we fix a function $\varkappa$ with the domain $\{0,1,2\}$ such that $\varkappa(0)=0, \varkappa(1)=1, \varkappa(2)=-1$. Then for the ternary expansion $n=1+\sum_{k=0}^{s} \gamma_{k} 3^{k}$ with $\gamma_{k} \in\{0,1,2\}$ we get $\psi_{n}=\pi / 2+$ $\sum_{k=0}^{s} \varkappa\left(\gamma_{k}\right) \delta_{k+1}$. From here it follows that

$$
\psi_{\gamma_{s} 3^{s}+\cdots+\gamma_{q} 3^{q}+k}=\psi_{k}+\Delta
$$

for $k=1,2, \ldots, 3^{q}$ with $\Delta=\sum_{j=q}^{s} \varkappa\left(\gamma_{j}\right) \delta_{j+1}$.

Given the points $\left(x_{k}\right)_{k=1}^{N+1}$, let $\omega_{N+1}(x)=\prod_{k=1}^{N+1}\left(x-x_{k}\right)$. For $k \leqq N+1$ we consider the fundamental Lagrange polynomial $l_{k, N}(x)=\frac{\omega_{N+1}(x)}{\left(x-x_{k}\right) \omega_{N+1}^{\prime}\left(x_{k}\right)}$. Then the Lebesgue function $\lambda_{N}(X ; x)$ is given as $\sum_{k=1}^{N+1}\left|l_{k, N}(x)\right|$ and the 
quantity $\Lambda_{N}(X)=\sup _{|x| \leqq 1} \lambda_{N}(X ; x)$ is called the Lebesgue constant of order $N$ of the sequence $X$. Clearly, $\Lambda_{N}(X)$ gives the norm of the Newton interpolating operator

$$
\mathcal{N}_{N}: C[-1,1] \longrightarrow \Pi_{N}: f \mapsto \sum_{k=1}^{N+1} f\left(x_{k}\right) l_{k, N}(x) .
$$

Here and subsequently, $\Pi_{N}$ denotes the set of all algebraic polynomials of degree at most $N$.

The aim of this paper is to estimate the growth of the sequence

$$
\left(\Lambda_{N}(X)\right)_{N=0}^{\infty} .
$$

Given $N$ with $3^{s}<N<3^{s+1}$ we will consider the even trigonometric polynomials

$\mu_{N}(\theta)=\omega_{N}(\cos \theta)=\prod_{k=1}^{N}\left[\cos \theta-\cos \psi_{k}\right]$ and $\rho_{N}(\theta)=\prod_{k=N+1}^{3^{s+1}}\left[\cos \theta-\cos \psi_{k}\right]$.

Thus, $\mu_{N}(\theta) \cdot \rho_{N}(\theta)=\tilde{T}_{3^{s+1}}(\cos \theta)$, where $\tilde{T}_{n}$ stands for the monic Chebyshev polynomials $2^{1-n} T_{n}$.

Given $\delta$, we define for any $n \in \mathbb{N}$ the polynomial

$$
t_{n}(\theta, \delta)=\prod_{k=1}^{n}\left[\cos \theta-\cos \left(\theta_{k}+\delta\right)\right]
$$

Here $\theta_{k}=\frac{2 k-1}{n} \frac{\pi}{2}$. We will restrict our attention to the values of the parameter $\delta$ with $|\delta|<\theta_{1}=\frac{\pi}{2 n}$. We see that the zeros of $t_{n}$ are defined by the angles corresponding to the Chebyshev polynomial $T_{n}$, but shifted by $\delta$.

For any even trigonometric polynomial $t(\theta)=\prod_{k=1}^{n}\left[\cos \theta-\cos \alpha_{k}\right]$ and $\delta \in \mathbb{R}$, let $S_{\delta} t$ denote the polynomial $\prod_{k=1}^{n}\left[\cos \theta-\cos \left(\alpha_{k}+\delta\right)\right]$. Thus, $t_{n}(\theta, \delta)=S_{\delta} \tilde{T}_{n}(\cos \theta)$.

\section{On the maximal value of $\left|\mu_{N}\right|$}

Let us show that $\left|\mu_{N}\right|$ attains its maximum at 0 .

Lemma 1 . Given $m \leqq n-1$ and $|\delta|<\frac{\pi}{2 n}$, let $0 \leqq \alpha \leqq \pi-\frac{m}{n} \pi$. Then

$$
\frac{t_{n}(\alpha, \delta)}{t_{n}(\alpha+m \pi / n, \delta)}=(-1)^{m} \prod_{k=1}^{m} \frac{\sin \left(\alpha+\theta_{k}\right)+\sin \delta}{\sin \left(\alpha+\theta_{k}\right)-\sin \delta} .
$$


Here for $\alpha=\theta_{1}+\delta, \ldots, \theta_{n-m}+\delta$ the fraction above is of the form $0 / 0$, so we consider it to be the corresponding limit.

PROOF. Here,

$$
t_{n}(\alpha, \delta)=\prod_{k=1}^{n}\left[\cos \alpha-\cos \left(\theta_{k}+\delta\right)\right]=(-2)^{n} \cdot \Pi_{1}(\alpha) \cdot \Pi_{2}(\alpha)
$$

with

$$
\Pi_{1}(\alpha)=\prod_{k=1}^{n} \sin \frac{1}{2}\left(\alpha+\frac{2 k-1}{2 n} \pi+\delta\right)
$$

and

$$
\Pi_{2}(\alpha)=\prod_{k=1}^{n} \sin \frac{1}{2}\left(\alpha-\frac{2 k-1}{2 n} \pi-\delta\right) .
$$

Similarly, $t_{n}(\alpha+m \pi / n, \delta)=(-2)^{n} \cdot \Pi_{1}(\alpha+m \pi / n) \cdot \Pi_{2}(\alpha+m \pi / n)$ with

$$
\begin{aligned}
& \Pi_{1}(\alpha+m \pi / n)=\prod_{k=1}^{n} \sin \frac{1}{2}\left(\alpha+\frac{2 k+2 m-1}{2 n} \pi+\delta\right), \\
& \Pi_{2}(\alpha+m \pi / n)=\prod_{k=1}^{n} \sin \frac{1}{2}\left(\alpha+\frac{2 m-2 k+1}{2 n} \pi-\delta\right) .
\end{aligned}
$$

After cancellation of common terms we get

$$
\begin{gathered}
\frac{t_{n}(\alpha, \delta)}{t_{n}(\alpha+m \pi / n, \delta)}=\frac{\sin \frac{1}{2}\left(\alpha+\frac{\pi}{2 n}+\delta\right) \cdots \sin \frac{1}{2}\left(\alpha+\frac{2 m-1}{2 n} \pi+\delta\right)}{\sin \frac{1}{2}\left(\alpha+\frac{2 n+1}{2 n} \pi+\delta\right) \cdots \sin \frac{1}{2}\left(\alpha+\frac{2 m+2 n-1}{2 n} \pi+\delta\right)} \\
\cdot \frac{\sin \frac{1}{2}\left(\alpha-\frac{2 n-2 m+1}{2 n} \pi-\delta\right) \cdots \sin \frac{1}{2}\left(\alpha-\frac{2 n-1}{2 n} \pi-\delta\right)}{\sin \frac{1}{2}\left(\alpha+\frac{2 m-1}{2 n} \pi-\delta\right) \cdots \sin \frac{1}{2}\left(\alpha+\frac{\pi}{2 n}-\delta\right)} \\
=\prod_{k=1}^{m} \frac{\sin \frac{1}{2}\left(\alpha+\frac{2 k-1}{2 n} \pi+\delta\right) \cdot \sin \frac{1}{2}\left(\alpha-\frac{2 n-2 k+1}{2 n} \pi-\delta\right)}{\sin \left(\alpha+\frac{2 k-1}{2 n} \pi-\delta\right) \cdot \sin \frac{1}{2}\left(\alpha+\frac{2 n+2 k-1}{2 n} \pi+\delta\right)} \\
=\prod_{k=1}^{m} \frac{\cos \left(\frac{\pi}{2}+\delta\right)-\cos \left(\frac{\pi}{2}-\alpha-\theta_{k}\right)}{\cos \left(\frac{\pi}{2}+\delta\right)-\cos \left(\frac{\pi}{2}+\alpha+\theta_{k}\right)}
\end{gathered}
$$

which gives the desired result. 
Lemma 2. Suppose $0<\alpha_{1} \leqq \alpha_{2} \leqq \cdots \leqq \alpha_{n}<\pi$ is such that the polynomial $t(\theta)=\prod_{k=1}^{n}\left[\cos \theta-\cos \alpha_{k}\right]$ attains its maximum modulus at $\theta=0$. If $0 \leqq \delta \leqq \pi-\alpha_{n}$, then $S_{\delta} t$ as well attains its maximum modulus at $\theta=0$.

ProOF. We proceed by induction. The polynomial $t_{1}(\theta)=\cos \theta-\cos \alpha_{1}$ attains its maximum modulus at $\theta=0$ if and only if $\pi / 2 \leqq \alpha_{1} \leqq \pi$. In this case $S_{\delta} t$ as well attains its maximum modulus at 0 . Suppose the statement is valid for any polynomial $t_{n}$ of the above type. Assume that for $t_{n+1}(\theta)=\prod_{k=1}^{n+1}\left[\cos \theta-\cos \alpha_{k}\right]$ with $0<\alpha_{1} \leqq \cdots \leqq \alpha_{n+1}<\pi$ we have $t_{n+1}(0) \geqq\left|t_{n+1}(\theta)\right|$ for any $0 \leqq \theta \leqq \pi$. Then $\pi / 2 \leqq \alpha_{n+1}$, since otherwise $\left|t_{n+1}(\pi)\right|>t_{n+1}(0)$. Here, $t_{n+1}(\theta)=t_{n}(\theta)\left[\cos \theta-\cos \alpha_{n+1}\right]$ and $S_{\delta} t_{n+1}(0)=$ $S_{\delta} t_{n}(0) \cdot\left[1-\cos \left(\alpha_{n+1}+\delta\right)\right] \geqq\left|S_{\delta} t_{n}(\theta)\right| \cdot\left|\cos \theta-\cos \left(\alpha_{n+1}+\delta\right)\right|$ for any $\theta$.

In the next lemma we localize the extrema of $t_{n}(\theta, \delta)$. For $|\delta|<\frac{\pi}{2 n}$ the function $\left|t_{n}(\cdot, \delta)\right|$ has $n+1$ local maxima on $[0, \pi]: \varphi_{0}=0, \varphi_{j} \in\left(\theta_{j}+\delta\right.$, $\left.\theta_{j+1}+\delta\right)$ for $j=1,2, \ldots, n-1$ and $\varphi_{n}=\pi$.

Lemma 3. Suppose $|\delta|<\frac{\pi}{2 n}$. For $j=1,2, \ldots, n-1$ we have $j \pi / n<\varphi_{j}<$ $j \pi / n+\delta$ if $\delta>0$, and $j \pi / n+\delta<\varphi_{j}<j \pi / n$ if $\delta<0$.

Proof. Without loss of generality we can assume $\delta>0$. Let $g(\theta)=$ $\sum_{k=1}^{n}\left[\cos \theta-\cos \left(\theta_{k}+\delta\right)\right]^{-1}$. Then $t_{n}^{\prime}(\theta, \delta)=-t_{n}(\theta, \delta) g(\theta) \sin \theta$. Let us show that

$$
g(j \pi / n)<0, g(j \pi / n+\delta)>0 \text { for } 1 \leqq j \leqq n-1 .
$$

This gives a change of sign of the derivative and a local extremal value of $t_{n}$ on the interval $(j \pi / n, j \pi / n+\delta)$.

We will use the representations

$$
\begin{gathered}
{[\cos u-\cos v]^{-1}=\frac{1}{2} \csc u\left[\cot \frac{v-u}{2}-\cot \frac{v+u}{2}\right]} \\
=\frac{1}{2} \csc v\left[\cot \frac{v-u}{2}+\cot \frac{v+u}{2}\right] .
\end{gathered}
$$

For $0 \leqq a \leqq \delta$ we have

$$
\begin{gathered}
g(j \pi / n+a)=\sum_{k=j+1}^{n}\left[\cos (j \pi / n+a)-\cos \left(\theta_{k}+\delta\right)\right]^{-1} \\
-\sum_{k=1}^{j}\left[\cos \left(\theta_{k}+\delta\right)-\cos (j \pi / n+a)\right]^{-1} .
\end{gathered}
$$


We represent the terms of the first sum as a difference of the corresponding cotangents, whereas for the second terms we use the second representation. Then

(3)

$$
g(j \pi / n+a) \cdot 2 \sin (j \pi / n+a)
$$

$$
\begin{gathered}
=\sum_{k=j+1}^{n}\left[\cot \left(\frac{2 k-2 j-1}{4 n} \pi+\frac{\delta-a}{2}\right)-\cot \left(\frac{2 k+2 j-1}{4 n} \pi+\frac{\delta+a}{2}\right)\right] \\
-\sum_{k=1}^{j}\left[\cot \left(\frac{2 k+2 j-1}{4 n} \pi+\frac{\delta+a}{2}\right)+\cot \left(\frac{2 j-2 k+1}{4 n} \pi-\frac{\delta-a}{2}\right)\right] \\
=\sum_{i=1}^{n-j} \cot \left(\frac{2 i-1}{4 n} \pi+\frac{\delta-a}{2}\right)-\sum_{i=1}^{j} \cot \left(\frac{2 i-1}{4 n} \pi-\frac{\delta-a}{2}\right) \\
-\sum_{i=j+1}^{n+j} \cot \left(\frac{2 i-1}{4 n} \pi+\frac{\delta+a}{2}\right) .
\end{gathered}
$$

Choose $a=0$. In the case $2 j<n$ we cancel common terms in the first and the third sums above. Here and in what follows we use the formula

$$
\sum_{i=n-j+1}^{n+j} \cot \left(\frac{2 i-1}{4 n} \pi+c\right)=\sum_{i=1}^{j}\left[\tan \left(\frac{2 i-1}{4 n} \pi-c\right)-\tan \left(\frac{2 i-1}{4 n} \pi+c\right)\right] \text {. }
$$

In this way,

$$
\begin{aligned}
g(j \pi / n) \cdot 2 & \sin (j \pi / n)=\sum_{i=1}^{j}\left[\cot \left(\frac{2 i-1}{4 n} \pi+\frac{\delta}{2}\right)-\cot \left(\frac{2 i-1}{4 n} \pi-\frac{\delta}{2}\right)\right] \\
& +\sum_{i=1}^{j}\left[\tan \left(\frac{2 i-1}{4 n} \pi+\frac{\delta}{2}\right)-\tan \left(\frac{2 i-1}{4 n} \pi-\frac{\delta}{2}\right)\right] \\
= & \sum_{i=1}^{j}\left[2 \csc \left(\frac{2 i-1}{2 n} \pi+\delta\right)-2 \csc \left(\frac{2 i-1}{2 n} \pi-\delta\right)\right] .
\end{aligned}
$$

The last expression is negative for positive $\delta$.

If $2 j \geqq n$ then arguing as above we get for $g(j \pi / n) \cdot 2 \sin (j \pi / n)$ the similar negative form

$$
\sum_{i=1}^{n-j}\left[2 \csc \left(\frac{2 i-1}{2 n} \pi+\delta\right)-2 \csc \left(\frac{2 i-1}{2 n} \pi-\delta\right)\right] .
$$


This proves the first inequality in (2).

We now turn to the case $a=\delta$ in (3). If $2 j<n$ then

$$
\begin{gathered}
g(j \pi / n+\delta) \cdot 2 \sin (j \pi / n+\delta)=\sum_{i=j+1}^{n-j}\left[\cot \frac{2 i-1}{4 n} \pi-\cot \left(\frac{2 i-1}{4 n} \pi+\delta\right)\right] \\
+\sum_{i=1}^{j}\left[\tan \left(\frac{2 i-1}{4 n} \pi+\delta\right)-\tan \left(\frac{2 i-1}{4 n} \pi-\delta\right)\right]
\end{gathered}
$$

which is positive for $\delta>0$.

For $2 j \geqq n$ we get

$$
\begin{gathered}
g(j \pi / n+\delta) \cdot 2 \sin (j \pi / n+\delta) \\
=\sum_{i=n-j+1}^{j}\left[\cot \left(\frac{2 i-1}{4 n} \pi-\delta\right)-\cot \left(\frac{2 i-1}{4 n} \pi\right)\right] \\
+\sum_{i=1}^{n-j}\left[\tan \left(\frac{2 i-1}{4 n} \pi+\delta\right)-\tan \left(\frac{2 i-1}{4 n} \pi-\delta\right)\right]>0,
\end{gathered}
$$

since for $j \leqq n-1$ all arguments of the cotangents above are positive. This gives (2) and completes the proof.

Lemma 4. Suppose $|\delta|<\frac{\pi}{2 n}$. If $\delta>0$ then the values $\left(\left|t_{n}\left(\varphi_{j}, \delta\right)\right|\right)_{j=0}^{n}$ decrease with respect to $j$. For $\delta<0$ we get an increasing set of values.

Proof. Suppose $\delta>0$. Let us fix $j$ with $1 \leqq j \leqq n$ and compare the values $\left|t_{n}\left(\varphi_{j}-\frac{\pi}{n}, \delta\right)\right|$ and $\left|t_{n}\left(\varphi_{j}, \delta\right)\right|$. By Lemma 1 ,

$$
\frac{\left|t_{n}\left(\varphi_{j}-\frac{\pi}{n}, \delta\right)\right|}{\left|t_{n}\left(\varphi_{j}, \delta\right)\right|}=\frac{\left|\sin \left(\varphi_{j}-\frac{\pi}{n}+\theta_{1}\right)+\sin \delta\right|}{\left|\sin \left(\varphi_{j}-\frac{\pi}{n}+\theta_{1}\right)-\sin \delta\right|} .
$$

By Lemma 3, we can consider the expression on the right without modulus. Clearly, the fraction derived is larger than 1 for $\delta>0$. Then $\left|t_{n}\left(\varphi_{j-1}, \delta\right)\right|$ $\geqq\left|t_{n}\left(\varphi_{j}-\frac{\pi}{n}, \delta\right)\right|$, since $\varphi_{j}-\frac{\pi}{n} \in\left(\theta_{j-1}+\delta, \theta_{j}+\delta\right)$ and $\varphi_{j-1}$ provides a maximal value of $\left|t_{n}(\cdot, \delta)\right|$ on this interval. From this, $\left|t_{n}\left(\varphi_{j-1}, \delta\right)\right| \geqq\left|t_{n}\left(\varphi_{j}, \delta\right)\right|$.

Similar arguments apply to the case $\delta<0$.

The next lemma deals with the product of polynomials of type $t_{3^{k}}$.

Lemma 5. Let $t(\theta)=t_{3^{q}}\left(\theta, \delta_{q+1}+\lambda\right) \cdot t_{3^{p}}\left(\theta,-\delta_{q+1}+\lambda\right)$ with $0 \leqq p<q$, $|\lambda|<\frac{1}{2} \delta_{q+1}$. Then $t(0) \geqq|t(\theta)|$ for any $\theta$. 
Proof. The proof will be divided into four steps. The first three of them correspond to the case $p \geqq 1$.

1) We prove the inequality $|t(\alpha)| \geqq\left|t\left(\alpha+\delta_{p}\right)\right|$ for $0 \leqq \alpha \leqq \pi-\delta_{p}$. This means that the function $|t(\cdot)|$ attains its maximum on the interval $\left[0, \delta_{p}\right]$.

2) For $0 \leqq \theta \leqq \beta:=\delta_{p}-\delta_{q+1}+\lambda$ we show $t(0) \geqq|t(\theta)|$. Thus it remains to consider only the values $\beta<\theta<\delta_{p}$.

3) We show that $t^{\prime}>0$ on $\left(\beta, \delta_{p}\right)$, so

$$
\max _{\left[\beta, \delta_{p}\right]}|t(\theta)|=\max \left\{|t(\beta)|,\left|t\left(\delta_{p}\right)\right|\right\} .
$$

Then $t(0) \geqq|t(\beta)|$, by 2$)$, and $t(0) \geqq\left|t\left(\delta_{p}\right)\right|$, by 1$)$.

4) We consider the case $p=0$ separately.

To prove 1 ), let us fix $0 \leqq \alpha \leqq \pi-\delta_{p}$. Since

$$
\frac{\sin u-\sin v}{\sin u+\sin v}=\frac{\tan \frac{u-v}{2}}{\tan \frac{u+v}{2}}
$$

we use Lemma 1 in the form

$$
\begin{gathered}
\frac{|t(\alpha)|}{\left|t\left(\alpha+\delta_{p}\right)\right|} \\
=\frac{\left|\tan \frac{1}{2}\left(\alpha+\theta_{1}^{(p)}-\delta_{q+1}+\lambda\right)\right|}{\left|\tan \frac{1}{2}\left(\alpha+\theta_{1}^{(p)}+\delta_{q+1}-\lambda\right)\right|} \cdot \prod_{k=1}^{3^{q-p}} \frac{\left|\tan \frac{1}{2}\left(\alpha+\theta_{k}^{(q)}+\delta_{q+1}+\lambda\right)\right|}{\left|\tan \frac{1}{2}\left(\alpha+\theta_{k}^{(q)}-\delta_{q+1}-\lambda\right)\right|} .
\end{gathered}
$$

Here $\theta_{k}^{(q)}=\left(k-\frac{1}{2}\right) \delta_{q}$. Therefore, $\theta_{1}^{(p)}=\frac{1}{2} \delta_{p}=\frac{1}{2} 3^{q-p} \delta_{q}, \theta_{3^{q-p}}^{(q)}=\delta_{p}-\frac{1}{2} \delta_{q}$ and, as is easy to check, arguments of all tangents above belong to the interval $(0, \pi / 2)$, so we can remove the signs of modulus. Thus we want to prove the inequality

$$
\prod_{k=1}^{3^{q-p}} \frac{\tan \frac{1}{2}\left(\alpha+\theta_{k}^{(q)}+\delta_{q+1}+\lambda\right)}{\tan \frac{1}{2}\left(\alpha+\theta_{k}^{(q)}-\delta_{q+1}-\lambda\right)} \geqq \frac{\tan \frac{1}{2}\left(\alpha+\theta_{1}^{(p)}+\delta_{q+1}-\lambda\right)}{\tan \frac{1}{2}\left(\alpha+\theta_{1}^{(p)}-\delta_{q+1}+\lambda\right)} .
$$

Let us consider the function $f(x)=\frac{\tan (A+x)}{\tan (A-x)}$ for

$$
0<x<A \quad \text { with } \quad x+A<\pi / 2 .
$$

One can check that $f$ increases under these conditions. 
The values $A=\frac{1}{2}\left(\alpha+\theta_{k}^{(q)}\right), x=\frac{1}{2}\left(\delta_{q+1}+\lambda\right)$ satisfy (5) for $k=1,2, \ldots$, $3^{q-p}$, therefore the left fraction in (4) attains its minimal value when $\lambda$ is minimal. Similarly, we can take $A=\frac{1}{2}\left(\alpha+\theta_{1}^{(p)}\right)$ and $x=\frac{1}{2}\left(\delta_{q+1}-\lambda\right)$, also satisfying (5). Then the right fraction in (4) attains its maximal value when $x$ is maximal, that is $\lambda$ is minimal. Therefore it is enough to prove (4) for $\lambda=-\frac{1}{2} \delta_{q+1}$. Set $\sigma:=\frac{1}{2} \delta_{q+1}$. We want to show

$$
\prod_{k=1}^{3^{q-p}} \frac{\tan \frac{1}{2}\left(\alpha+\theta_{k}^{(q)}+\sigma\right)}{\tan \frac{1}{2}\left(\alpha+\theta_{k}^{(q)}-\sigma\right)} \geqq \frac{\tan \frac{1}{2}\left(\alpha+\theta_{1}^{(p)}+3 \sigma\right)}{\tan \frac{1}{2}\left(\alpha+\theta_{1}^{(p)}-3 \sigma\right)},
$$

which is equivalent to

$$
\prod_{k=1}^{3^{q-p}}\left[1+\frac{2 \sin \sigma}{\sin \left(\alpha+\theta_{k}^{(q)}\right)-\sin \sigma}\right] \geqq 1+\frac{2 \sin 3 \sigma}{\sin \left(\alpha+\theta_{1}^{(p)}\right)-\sin 3 \sigma} .
$$

We can restrict ourselves in the product on the left only to the values $k=1, k=\frac{1}{2}\left(3^{q-p}+1\right)$ and $k=3^{q-p}$, since all terms in this product exceed 1 . Here $\theta_{\left(3^{q-p}+1\right) / 2}^{(q)}=\theta_{1}^{(p)}=\frac{1}{2} \delta_{p}$. We use the following notations:

$$
\begin{gathered}
b_{1}=\sin \left(\alpha+\frac{1}{2} \delta_{q}\right)-\sin \sigma, \quad b_{2}=\sin \left(\alpha+\frac{1}{2} \delta_{p}\right)-\sin \sigma \\
b_{3}=\sin \left(\alpha+\delta_{p}-\frac{1}{2} \delta_{q}\right)-\sin \sigma, \quad a=\sin \left(\alpha+\frac{1}{2} \delta_{p}\right)-\sin 3 \sigma .
\end{gathered}
$$

Then the desired inequality takes the form

$$
\prod_{k=1}^{3}\left(1+\frac{2 \sin \sigma}{b_{k}}\right) \geqq 1+\frac{2 \sin 3 \sigma}{a} .
$$

We multiply all terms on the left, neglect the term containing $\sin ^{3} \sigma$, and use the inequality $\sin 3 \sigma<3 \sin \sigma$ in order to replace the desired inequality by

$$
\frac{1}{b_{1}}+\frac{1}{b_{2}}+\frac{1}{b_{3}}+2 \sin \sigma\left(\frac{1}{b_{1} b_{2}}+\frac{1}{b_{1} b_{3}}+\frac{1}{b_{2} b_{3}}\right) \geqq \frac{3}{a} .
$$

The last inequality is equivalent to

$$
a\left(b_{1} b_{2}+b_{1} b_{3}+b_{2} b_{3}\right)+2 a \sin \sigma\left(b_{1}+b_{2}+b_{3}\right) \geqq 3 b_{1} b_{2} b_{3} .
$$


We express both parts of the inequality above as functions of the variable $y:=\sin \left(\alpha+\frac{1}{2} \delta_{p}\right)$. Clearly, $y>0$. Also for brevity, let $\mu:=\frac{1}{2}\left(\delta_{p}-\delta_{q}\right)$. Then $b_{2}=y-\sin \sigma, a=y-\sin 3 \sigma, b_{1}+b_{3}=2 y \cos \mu-2 \sin \sigma$. Therefore, $b_{1}+b_{2}$ $+b_{3}=y(2 \cos \mu+1)-3 \sin \sigma$. Also,

$$
b_{1} b_{3}=y^{2}-2 y \sin \sigma \cos \mu+\sin ^{2} \sigma-\sin ^{2} \mu
$$

and

$b_{2}\left(b_{1}+b_{3}\right)+b_{1} b_{3}=y^{2}(2 \cos \mu+1)-2 y \sin \sigma(2 \cos \mu+1)+3 \sin ^{2} \sigma-\sin ^{2} \mu$.

The left part of (6) can be written in new terms as $A_{3} y^{3}+A_{2} y^{2}+A_{1} y$ $+A_{0}$ with $A_{3}=2 \cos \mu+1, A_{2}=-\sin 3 \sigma(2 \cos \mu+1), A_{1}=-3 \sin ^{2} \sigma-\sin ^{2} \mu$, and $A_{0}=\sin 3 \sigma\left(3 \sin ^{2} \sigma+\sin ^{2} \mu\right)$. On the other hand, on the right we have $B_{3} y^{3}+B_{2} y^{2}+B_{1} y+B_{0}$ with $B_{3}=3, B_{2}=-3 \sin \sigma(2 \cos \mu+1), B_{1}=$ $3\left[\sin ^{2} \sigma(2 \cos \mu+1)-\sin ^{2} \mu\right], B_{0}=-3 \sin \sigma\left(\sin ^{2} \sigma-\sin ^{2} \mu\right)$. We transfer $A_{3} y^{3}$ to the right, whereas $B_{2} y^{2}+B_{1} y+B_{0}$ to the left side of (6), so we reduce the inequality to the following: $\left(A_{2}-B_{2}\right) y^{2}+\left(A_{1}-B_{1}\right) y+A_{0}-B_{0}$ $\geqq\left(B_{3}-A_{3}\right) y^{3}$, that is

$$
\begin{gathered}
4 \sin ^{3} \sigma(2 \cos \mu+1) \cdot y^{2}+\left(2 \sin ^{2} \mu-12 \sin ^{2} \sigma \cos ^{2} \frac{\mu}{2}\right) \cdot y \\
+4 \sin ^{3} \sigma\left(3 \cos ^{2} \sigma-\sin ^{2} \mu\right) \geqq 4 \sin ^{2} \frac{\mu}{2} \cdot y^{3} .
\end{gathered}
$$

The coefficient of $y^{2}$ is positive. Also, $\sigma=\frac{\pi}{2 \cdot 3^{q+1}} \leqq \frac{\pi}{54}$, as $q \geqq 2$. Therefore $3 \cos ^{2} \sigma>\sin ^{2} \mu$. Since $0<y<1$, it remains to show that

$$
2 \sin ^{2} \mu-12 \sin ^{2} \sigma \cos ^{2} \frac{\mu}{2} \geqq 4 \sin ^{2} \frac{\mu}{2},
$$

which can be reduced to $\tan ^{2} \frac{\mu}{2} \cos \mu \geqq 3 \sin ^{2} \sigma$.

Here, $\mu \geqq \frac{1}{2}\left(\delta_{q-1}-\delta_{q}\right)=6 \sigma$. It follows that $\tan ^{2} \frac{\mu}{2} \geqq 9 \sigma^{2}$. On the other hand, $\mu<\frac{1}{2} \delta_{p} \leqq \pi / 6$, which gives the desired inequality and completes the proof of the first step.

To prove 2), let us denote by $\varphi_{1}^{(p)}$ the point on the interval

$$
\left(\theta_{1}^{(p)}-\delta_{q+1}+\lambda, \theta_{2}^{(p)}-\delta_{q+1}+\lambda\right)
$$

where $t_{3^{p}}$ attains its minimal value. By Lemma $3, \beta=\delta_{p}-\delta_{q+1}+\lambda<\varphi_{1}^{(p)}$ $<\delta_{p}$. Therefore, the polynomial $t_{3^{p}}$ decreases on the interval $[0, \beta]$. Also, by 
Lemma 4 , the values $\left|t_{3^{q}}\left(\varphi_{j}^{(q)}, \delta_{q+1}+\lambda\right)\right|$ of the corresponding local maxima decrease as $j$ increases. Therefore, $t(0) \geqq|t(\theta)|$ for $0 \leqq \theta \leqq \beta$.

$3)$ We turn to the values $\theta \in I:=\left(\beta, \delta_{p}\right)$. We cannot apply the arguments of Lemma 1 to this case. For example, if $\lambda \succsim-\sigma$ and $\alpha \succsim-3 \sigma=-\theta_{1}^{(q)}$, then $\alpha+\delta_{p} \in I$. One can show that $t(\alpha)<\left|t\left(\alpha+\delta_{p}\right)\right|$ here, since the value $t_{3^{q}}\left(\alpha, \delta_{q+1}+\lambda\right)$ is rather small.

Let us analyze the behavior of the polynomial $t$ on $I$ in detail. Clearly, the polynomial $t_{3^{p}}$ is negative here and, what is more, the values of $t_{3^{p}}$ on $I$ are near to its local minimum. On the other hand, the polynomial $t_{3^{q}}$ has a root at the point $z:=\delta_{p}-\frac{1}{2} \delta_{q+1}+\lambda \in I$, furthermore this is a root with odd multiplicity $3^{q-p}$. Therefore, $t_{3^{q}}$ changes its sign from plus to minus when $\theta$ passes this point. Thus, $t<0$ on $(\beta, z)$ and $t>0$ on $\left(z, \delta_{p}\right)$. Let us show that $t^{\prime}>0$ on $I$. We have $t_{3^{p}}^{\prime}(\theta)=-t_{3^{p}}(\theta) g_{p}(\theta) \sin \theta, t_{3^{q}}^{\prime}(\theta)=-t_{3^{q}}(\theta) g_{q}(\theta) \sin \theta$, where

$$
\begin{aligned}
& g_{p}(\theta)=\sum_{k=1}^{3^{p}}\left[\cos \theta-\cos \left(\theta_{k}^{(p)}-\delta_{q+1}+\lambda\right)\right]^{-1}, \\
& g_{q}(\theta)=\sum_{k=1}^{3^{q}}\left[\cos \theta-\cos \left(\theta_{k}^{(q)}+\delta_{q+1}+\lambda\right)\right]^{-1} .
\end{aligned}
$$

Hence, $t^{\prime}(\theta)=-t(\theta) g(\theta) \sin \theta$ with $g=g_{p}+g_{q}$. Note that the functions $g_{q}$ and $g$ have a vertical asymptote at $z$. We see that the inequalities $g>0$ on $(\beta, z)$ and $g<0$ on $\left(z, \delta_{p}\right)$ will give the positivity of $t^{\prime}$ on $I$.

The function $f(x)=\sum_{k=1}^{n} \frac{1}{x-x_{k}}$ decreases on any interval of its continuity. Therefore the function $f(\cos \theta)$ increases on any such interval and it is enough to show that

$$
g\left(\delta_{p}\right)<0 \text { and } g(\beta)>0 .
$$

Let us first examine the value $\delta_{p}$. Arguing as in Lemma 3 (for the case $j=1, a=0)$, we get

$$
g_{p}\left(\delta_{p}\right) \cdot \sin \delta_{p}=\csc \left(\frac{1}{2} \delta_{p}-\delta_{q+1}+\lambda\right)-\csc \left(\frac{1}{2} \delta_{p}+\delta_{q+1}-\lambda\right) .
$$

Similarly,

$$
g_{q}\left(\delta_{p}\right) \cdot \sin \delta_{p}=\sum_{k=1}^{3^{q-p}}\left[\csc \left(\theta_{k}^{(q)}+\delta_{q+1}+\lambda\right)-\csc \left(\theta_{k}^{(q)}-\delta_{q+1}-\lambda\right)\right] .
$$


In order to get the first inequality in (7), we need to show that

$$
\begin{aligned}
& \csc \left(\theta_{1}^{(p)}-\delta_{q+1}+\lambda\right)+\sum_{k=1}^{3^{q-p}} \csc \left(\theta_{k}^{(q)}+\delta_{q+1}+\lambda\right) \\
& <\csc \left(\theta_{1}^{(p)}+\delta_{q+1}-\lambda\right)+\sum_{k=1}^{3^{q-p}} \csc \left(\theta_{k}^{(q)}-\delta_{q+1}-\lambda\right) .
\end{aligned}
$$

Since the left part of this inequality increases, whereas the right part decreases, when $\lambda$ becomes smaller, it is enough to consider the value $\lambda=$ $-\frac{1}{2} \delta_{q+1}=-\sigma$. We want to show

(8) $\sum_{k=1}^{3^{q-p}}\left[\csc \left(\theta_{k}^{(q)}+\sigma\right)-\csc \left(\theta_{k}^{(q)}-\sigma\right)\right]<\csc \left(\theta_{1}^{(p)}+3 \sigma\right)-\csc \left(\theta_{1}^{(p)}-3 \sigma\right)$.

The function $f(x)=\csc (x+3 \sigma)-\csc (x-3 \sigma)$ increases as $x$ increases with $3 \sigma<x<\pi-3 \sigma$. Therefore it suffices to consider the smallest value of $\theta_{1}^{(p)}$ that is the case $q=p+1$, when $\theta_{1}^{(p)}=9 \sigma$. On the other hand, we can take only the first term in the sum in (8), since all expressions in the square brackets there are negative. Thus, for $\theta_{1}^{(q)}=3 \sigma$, we reduce ( 8 ) to the inequality

$$
\csc (4 \sigma)-\csc (2 \sigma)<\csc (12 \sigma)-\csc (6 \sigma),
$$

which is valid for $0<\sigma \leqq \frac{\pi}{54}$, as is easy to check. Thus, $g\left(\delta_{p}\right)<0$.

Our next goal is to show the second inequality in (7). In the same manner we get the representation

$$
\begin{gathered}
g_{p}(\beta) \cdot 2 \sin \beta=\sum_{k=1}^{3^{p}-2}\left[\cot \left(\frac{2 k+1}{4} \delta_{p}\right)-\cot \left(\frac{2 k+1}{4} \delta_{p}-\delta_{q+1}+\lambda\right)\right] \\
+\tan \left(\frac{\delta_{p}}{4}-\delta_{q+1}+\lambda\right)-\tan \left(\frac{\delta_{p}}{4}+\delta_{q+1}-\lambda\right) .
\end{gathered}
$$

This value is negative and, as a function of $\lambda$ with $|\lambda|<\sigma$, it attains its minimum when $\lambda=-\sigma$. Therefore,

$$
\begin{aligned}
g_{p}(\beta) & \cdot 2 \sin \beta \geqq-\frac{\sin 6 \sigma}{\cos \left(\frac{\delta_{p}}{4}-3 \sigma\right) \cdot \cos \left(\frac{\delta_{p}}{4}+3 \sigma\right)} \\
& +\sum_{k=1}^{3^{p}-2} \frac{-\sin 3 \sigma}{\sin \frac{2 k+1}{4} \delta_{p} \cdot \sin \left(\frac{2 k+1}{4} \delta_{p}-3 \sigma\right)} .
\end{aligned}
$$


The term $g_{q}(\beta)$ can be handled in the same way. We get

$$
\begin{gathered}
g_{q}(\beta)=\sum_{k=3^{q-p}}^{3^{q}}\left[\cos \left(3^{q-p} \delta_{q}-2 \sigma+\lambda\right)-\cos \left(\left(k-\frac{1}{2}\right) \delta_{q}+2 \sigma+\lambda\right)\right]^{-1} \\
-\sum_{k=1}^{3^{q-p}-1}\left[\cos \left(\left(k-\frac{1}{2}\right) \delta_{q}+2 \sigma+\lambda\right)-\cos \left(3^{q-p} \delta_{q}-2 \sigma+\lambda\right)\right]^{-1} .
\end{gathered}
$$

As in Lemma 3, the terms of the first (second) sum can be represented as a difference (a sum) of the corresponding cotangents. Hence, $g_{q}(\beta) \cdot 2 \sin \beta$ $=A_{1}-A_{2}-B_{1}-B_{2}$, where

$$
\begin{gathered}
A_{1}=\sum_{k=1}^{3^{q}-3^{q-p}+1} \cot \frac{1}{2}(6 k-5) \sigma, \quad A_{2}=\sum_{k=1}^{3^{q}-3^{q-p}+1} \cot \frac{1}{2}\left(2 \delta_{p}+6 k \sigma-9 \sigma+2 \lambda\right), \\
B_{1}=\sum_{k=1}^{3^{q-p}-1} \cot \frac{1}{2}(6 k-1) \sigma, \quad B_{2}=\sum_{k=1}^{3^{q-p}-1} \cot \frac{1}{2}\left(\delta_{p}+6 k \sigma-3 \sigma+2 \lambda\right) .
\end{gathered}
$$

We decompose $A_{1}$ in the sum

$$
A_{11}+A_{12}+A_{13}=\sum_{k=1}^{3^{q-p}-1}+\sum_{k=3^{q-p}}^{2 \cdot 3^{q-p}-1}+\sum_{k=2 \cdot 3^{q-p}}^{3^{q}-3^{q-p}+1} .
$$

Also,

$$
A_{2}=A_{21}+A_{22}=\sum_{k=1}^{3^{q}-3 \cdot 3^{q-p}+2}+\sum_{k=3^{q}-3 \cdot 3^{q-p}+3}^{3^{q}-3^{q-p}+1} .
$$

Here the difference

$$
\begin{aligned}
A_{13}-A_{21} & =\sum_{k=1}^{3^{q}-3 \cdot 3^{q-p}+2}\left[\cot \frac{1}{2}\left(2 \delta_{p}+6 k \sigma-11 \sigma\right)\right. \\
& \left.-\cot \frac{1}{2}\left(2 \delta_{p}+6 k \sigma-9 \sigma+2 \lambda\right)\right]
\end{aligned}
$$

is positive, since $|\lambda|<\sigma$. We want to estimate $g_{q}(\beta)$ from below, so we can neglect this difference. Also,

$$
\begin{gathered}
A_{12}-B_{2}=\cot \frac{1}{2}\left(\delta_{p}-5 \sigma\right)+\sum_{k=1}^{3^{q-p}-1}\left[\cot \frac{1}{2}\left(\delta_{p}+(6 k-5) \sigma\right)\right. \\
\left.-\cot \frac{1}{2}\left(\delta_{p}+(6 k-3) \sigma+2 \lambda\right)\right]>\cot \frac{1}{2}\left(\delta_{p}-5 \sigma\right) .
\end{gathered}
$$


Therefore,

$$
\begin{aligned}
g_{q}(\beta) \cdot 2 \sin \beta>A_{11}-A_{22}-B_{1}+\cot \frac{1}{2}\left(\delta_{p}-5 \sigma\right) \\
=\sum_{k=1}^{3^{q-p}-1}\left[\cot \frac{1}{2}(6 k-5) \sigma-\cot \frac{1}{2}(6 k-1) \sigma\right] \\
+\sum_{k=1}^{3^{q-p}-1}\left[\tan \frac{1}{2}(6 k \sigma-3 \sigma+2 \lambda)-\tan \frac{1}{2}(6 k \sigma-3 \sigma-2 \lambda)\right] \\
+\cot \frac{1}{2}\left(\delta_{p}-5 \sigma\right)+\tan \frac{1}{2}\left(\delta_{p}-3 \sigma+2 \lambda\right) .
\end{aligned}
$$

The value $\lambda=-\sigma$ only reduces this expression. From here,

$$
g_{q}(\beta) \cdot 2 \sin \beta \geqq \sum_{k=1}^{3^{q-p}-1} \frac{4 \cos (6 k-3) \sigma \sin 2 \sigma}{\sin (6 k-5) \sigma \sin (6 k-1) \sigma}+\frac{2}{\sin \left(\delta_{p}-5 \sigma\right)} .
$$

Combining this with $(9)$ we get the desired inequality $g(\beta)>0$ provided

$$
\frac{2}{\sin \left(\delta_{p}-5 \sigma\right)}>\frac{\sin 6 \sigma}{\cos \left(\delta_{p} / 4-3 \sigma\right) \cdot \cos \left(\delta_{p} / 4+3 \sigma\right)}
$$

and

$$
\sum_{k=1}^{3^{q-p}-1} \frac{4 \cos (6 k-3) \sigma \sin 2 \sigma}{\sin (6 k-5) \sigma \sin (6 k-1) \sigma}>\sum_{k=1}^{3^{p}-2} \frac{\sin 3 \sigma}{\sin \frac{2 k+1}{4} \delta_{p} \cdot \sin \left(\frac{2 k+1}{4} \delta_{p}-3 \sigma\right)}
$$

It is a simple matter to check the first inequality for $0<\sigma \leqq \frac{\pi}{54}$ and $\delta_{p}$ with $18 \sigma=3 \delta_{q} \leqq \delta_{p} \leqq \pi / 3$. On the other hand, increase of $p$ will enlarge the right part of the second inequality and decrease the left one. Therefore we can assume $p=q-1$, that is $\delta_{p}=18 \sigma$. Moreover, we consider only the first term on the left. It remains to show that

$$
\frac{4 \cos 3 \sigma \sin 2 \sigma}{\sin \sigma \sin 5 \sigma}>\sum_{k=1}^{3^{p}-2} \frac{\sin 3 \sigma}{\sin \left(9 k+\frac{9}{2}\right) \sigma \sin \left(9 k+\frac{3}{2}\right) \sigma}
$$

For the sum on the right we have

$$
\sum_{k=1}^{3^{p}-2}<\sum_{k=1}^{3^{p}-2} \frac{3 \sigma}{\sin ^{2} 9 k \sigma}<\frac{\pi^{2}}{4} \sum_{k=1}^{3^{p}-2} \frac{3 \sigma}{81 k^{2} \sigma^{2}}<\frac{\pi^{4}}{648 \sigma}
$$


since $9 k \sigma<\pi / 2$. Eventually,

$$
\frac{4 \cos 3 \sigma \sin 2 \sigma}{\sin \sigma \sin 5 \sigma}>\frac{8 \cos 3 \sigma \cos \sigma}{5 \sigma}>\frac{2}{5 \sigma},
$$

which exceeds $\frac{\pi^{4}}{648 \sigma}$ for the given values of $\sigma$.

4) To deal with $p=0$, we note that

$$
t_{1}\left(\theta,-\delta_{q+1}+\lambda\right)=\cos \theta-\cos \left(\psi_{1}-\delta_{q+1}+\lambda\right)=\cos \theta-\sin \left(\delta_{q+1}-\lambda\right) .
$$

If $\theta \leqq \pi / 2$ then $\left|t_{1}(\theta)\right| \leqq t_{1}(0)$. Indeed, this is evident for $\theta \leqq \pi / 2-\delta_{q+1}+\lambda$. If $\pi / 2-\delta_{q+1}+\lambda<\theta \leqq \pi / 2$ then

$$
\left|t_{1}(\theta)\right| \leqq\left|t_{1}(\pi / 2)\right|=\sin \left(\delta_{q+1}-\lambda\right) \leqq t_{1}(0),
$$

since $2 \sin \left(\delta_{q+1}-\lambda\right) \leqq 2 \sin 3 \sigma \leqq 1$ for $\sigma=\frac{\pi}{2 \cdot 3^{q}} \leqq \frac{\pi}{18}$.

Furthermore, by Lemma 4 , the values of the local maxima of $\left|t_{3^{q}}\right|$ decrease, so $t(0) \geqq|t(\theta)|$ for $0 \leqq \theta \leqq \pi / 2$.

Let us fix $\theta>\pi / 2$. For $t_{1}$ we get

$$
\frac{t_{1}(0)}{\left|t_{1}(\theta)\right|}>\frac{1-\sin 3 \sigma}{-\cos \theta+\sin 3 \sigma} \geqq \frac{1-\sin 3 \sigma}{1+\sin 3 \sigma}>\frac{1-3 \sigma}{1+3 \sigma} \text {. }
$$

Given $\theta$, we choose $m$ such that

$$
\theta_{m}^{(q)}+\delta_{q+1}+\lambda \leqq \theta<\theta_{m+1}^{(q)}+\delta_{q+1}+\lambda
$$

Then $\left|t_{3^{q}}(\theta)\right| \leqq\left|t_{3^{q}}\left(\varphi_{m}\right)\right|$, where by Lemma $3, m \delta_{q}<\varphi_{m}<m \delta_{q}+\delta_{q+1}+\lambda$. Since $\theta>\pi / 2$, we have $m \geqq 2$. We compare, by means of Lemma 1 , the values of $\left|t_{3^{q}}(\cdot)\right|$ at the points $\varphi_{m}$ and $\alpha:=\varphi_{m}-m \delta_{q}$. Clearly, $0<\alpha<$ $\delta_{q+1}+\lambda<\theta_{1}^{(q)}$ and $t_{3^{q}}(0)>t_{3^{q}}(\alpha)>0$. Thus,

$$
\frac{t_{3^{q}}(0)}{\left|t_{3^{q}}(\theta)\right|} \geqq \frac{t_{3^{q}}(\alpha)}{\left|t_{3^{q}}(\theta)\right|}=\prod_{k=1}^{m} \frac{\sin \left(\alpha+\theta_{k}^{(q)}\right)+\sin \left(\delta_{q+1}+\lambda\right)}{\sin \left(\alpha+\theta_{k}^{(q)}\right)-\sin \left(\delta_{q+1}+\lambda\right)} .
$$

All fractions in the product exceed 1, so we can consider only the first two terms. Moreover, arguing as above, we see that the expression takes its minimal value when $\lambda$ is minimal, whereas $\alpha$ is maximal. Therefore,

$$
\frac{t_{3^{q}}(0)}{\left|t_{3^{q}}(\theta)\right|}>\prod_{k=1}^{2}\left(1+\frac{2 \sin \sigma}{\sin \left(\theta_{1}^{(q)}+\theta_{k}^{(q)}\right)-\sin \sigma}\right) .
$$



we get

Since $\theta_{1}^{(q)}=3 \sigma, \theta_{2}^{(q)}=9 \sigma$, and $\sin u+\sin v \geqq \sin (u+v)$ for $0 \leqq u, v \leqq \pi$,

$$
\frac{t_{3^{q}}(0)}{\left|t_{3^{q}}(\theta)\right|}>\left(1+\frac{2 \sin \sigma}{\sin 5 \sigma}\right)\left(1+\frac{2 \sin \sigma}{\sin 11 \sigma}\right)>1+2 \sin \sigma \cdot \frac{16}{55 \sigma} .
$$

Combining this with $(10)$ we see that $t(0)>|t(\theta)|$, provided

$$
\frac{16}{55} \frac{\sin \sigma}{\sigma} \geqq \frac{3 \sigma}{1-3 \sigma}
$$

The left part of this inequality decreases, whereas the right part is an increasing function, so we have to check the inequality only for maximal $\sigma$.

Suppose $q \geqq 2$. Then $0<\sigma \leqq \frac{\pi}{54}$ and $\frac{16 \cdot 54}{55 \cdot \pi} \sin \frac{\pi}{54} \geqq 0.29$. On the other hand, $\frac{\pi}{18-\pi}<0.22$, which proves the inequality.

We cannot apply the above mentioned arguments for $q=1$. However one can use in this case a direct computation. Here

$$
\begin{gathered}
t_{1}\left(\theta,-\delta_{2}+\lambda\right)=\cos \theta-\sin \left(\frac{\pi}{9}-\lambda\right) \\
t_{3}\left(\theta, \delta_{2}+\lambda\right)=\left[\cos \theta-\cos \left(\frac{\pi}{6}+\frac{\pi}{9}+\lambda\right)\right] \cdot\left[\cos \theta-\cos \left(\frac{\pi}{2}+\frac{\pi}{9}+\lambda\right)\right] \\
\cdot\left[\cos \theta-\cos \left(\frac{5 \pi}{6}+\frac{\pi}{9}+\lambda\right)\right]
\end{gathered}
$$

and we consider the values $|\lambda|<\frac{\pi}{18}$ and $\theta \in I:=\left[\frac{\pi}{2}, \pi\right]$. We see that diminution of $\lambda$ will decrease the value of $t=t_{1} t_{3}$ at $\theta=0$ and will increase the values of $|t|$ at its extremal points on $I$. Therefore, in the worst case $\lambda=-\frac{\pi}{18}$, we get

$$
t(\theta)=\left(\cos \theta-\frac{1}{2}\right)\left(\cos \theta-\cos \frac{2 \pi}{9}\right)\left(\cos \theta-\cos \frac{5 \pi}{9}\right)\left(\cos \theta-\cos \frac{8 \pi}{9}\right) .
$$

As before, $t^{\prime}(\theta)=-t(\theta) g(\theta) \sin \theta$, where

$$
\begin{aligned}
g(\theta) & =\left(\cos \theta-\cos \frac{2 \pi}{9}\right)^{-1}+\left(\cos \theta-\cos \frac{5 \pi}{9}\right)^{-1} \\
& +\left(\cos \theta-\cos \frac{8 \pi}{9}\right)^{-1}+\left(\cos \theta-\frac{1}{2}\right)^{-1} .
\end{aligned}
$$


We see that $t\left(\frac{\pi}{2}\right)>0$. Since $g\left(\frac{\pi}{2}\right) \approx 3.51>0$ and $t$ has only two zeros on $I$, the polynomial $t$ decreases on $\left[\frac{\pi}{2}, \varphi\right]$ and then increases on $[\varphi, \pi]$, where $\varphi$ is a point of minimum of $t$ with $\frac{5 \pi}{9}<\varphi<\frac{8 \pi}{9}$. Thus, it is enough to check only three inequalities: $t(0) \geqq t\left(\frac{\pi}{2}\right), t(0) \geqq t(\pi), t(0) \geqq|t(\varphi)|$. Direct computation gives $t(0) \approx 0.2663, t\left(\frac{\pi}{2}\right) \approx 0.0625, t(\pi) \approx 0.1320$. In order to get the third inequality, let us find a small enough interval $(a, b) \subset\left(\frac{5 \pi}{9}, \frac{8 \pi}{9}\right)$ such that $g(a) \cdot g(b)<0$, so $\varphi \in(a, b)$. If $a<\theta<b$ then, clearly,

$$
\begin{gathered}
|t(\theta)|<c:=\left(\frac{1}{2}-\cos b\right)\left(\cos \frac{2 \pi}{9}-\cos b\right) \\
\cdot\left(\cos \frac{5 \pi}{9}-\cos b\right)\left(\cos a-\cos \frac{8 \pi}{9}\right) .
\end{gathered}
$$

Now the values $a=\frac{52 \pi}{72}, b=\frac{53 \pi}{72}$ can be taken. Indeed, $g(a) \approx-0.3483, g(b)$ $\approx 0.2498$ and $c \approx 0.2526<t(0)$.

We see that $|t|$ attains its maximum value at 0 , just as in all previous cases.

The main result of this section is

Lemma 6 . For any $N \in \mathbb{N}, 0 \leqq \theta \leqq \pi$ we have $\mu_{N}(0) \geqq\left|\mu_{N}(\theta)\right|$.

Proof. If $N=3^{s}$ with $s \in \mathbb{N}_{0}$, then the function $\mu_{3^{s}}(\theta)=2^{1-3^{s}} \cos 3^{s} \theta$ attains its maximum modulus at 0 . Also, $\mu_{2}(\theta)=\cos \theta(\cos \theta-\cos 5 \pi / 6)$ has this property. Therefore we have the desired inequality for $1 \leqq N \leqq 3$. By induction, assume it is valid for $N \leqq 3^{s}$. Suppose $3^{s}<N<3^{s+1}$. If $N \leqq$ $2 \cdot 3^{s}$, that is $N=3^{s}+k$ for $1 \leqq k \leqq 3^{s}$, then $\psi_{N}=\psi_{k}+\delta_{s+1}$. By induction, $\mu_{k}(0) \geqq\left|\mu_{k}(\theta)\right|$ for all $\theta$, so, by Lemma $2, \mu_{N}(0) \geqq\left|\mu_{N}(\theta)\right|$ for all $\theta$.

Let us consider the remaining case $N=2 \cdot 3^{s}+\gamma_{r} \cdot 3^{r}+\cdots+\gamma_{q} \cdot 3^{q}+$ $\gamma_{p} \cdot 3^{p}$. Here $\gamma_{k} \neq 0$, that is $\gamma_{k}=1$ or $\gamma_{k}=2$, and $s>r>\cdots>q>p \geqq 0$. The first $3^{s}$ angles $\left(\psi_{k}\right)_{k=1}^{3^{s}}$ give us the polynomial $\mu_{3^{s}}$, the next $\left(\psi_{k}\right)_{k=3^{s}+1}^{2 \cdot 3^{s}}$ are zeros of $t_{3^{s}}\left(\cdot, \delta_{s+1}\right)$. For the numbers $\left(2 \cdot 3^{s}+k\right)_{k=1}^{3^{r}}$ we have the angles $\psi_{2.3^{s}+k}=\psi_{k}-\delta_{s+1}$ with $k=1, \ldots, 3^{r}$, and the corresponding polynomial $t_{3^{r}}\left(\cdot,-\delta_{s+1}\right)$. If $\gamma_{r}=2$ then for the next $3^{r}$ indices $\left(2 \cdot 3^{s}+3^{r}+k\right)_{k=1}^{3^{r}}$ we get the angles $\psi_{2 \cdot 3^{s}+3^{r}+k}=-\delta_{s+1}+\delta_{r+1}+\psi_{k}$ for $k=1, \ldots, 3^{r}$, and the corresponding polynomial $t_{3^{r}}\left(\cdot,-\delta_{s+1}+\delta_{r+1}\right)$. Therefore,

$$
\mu_{N}=\mu_{3^{s}} \cdot t_{3^{s}}\left(\cdot, \delta_{s+1}\right) \cdot t_{3^{r}}\left(\cdot,-\delta_{s+1}\right) \cdots \quad \text { for } \quad \gamma_{r}=1,
$$

and

$$
\mu_{N}=\mu_{3^{s}} \cdot t_{3^{s}}\left(\cdot, \delta_{s+1}\right) \cdot t_{3^{r}}\left(\cdot,-\delta_{s+1}\right) \cdot t_{3^{r}}\left(\cdot,-\delta_{s+1}+\delta_{r+1}\right) \cdots \quad \text { for } \quad \gamma_{r}=2 .
$$


Here $\cdots$ denotes the product of certain polynomials $t_{3^{k}}$ with $k<r$. Both functions $t_{3^{s}}\left(\cdot, \delta_{s+1}\right) \cdot t_{3^{r}}\left(\cdot,-\delta_{s+1}\right)$ and $t_{3^{r}}\left(\cdot,-\delta_{s+1}+\delta_{r+1}\right)$ have maximum modulus at 0 , the first by Lemma 5 , and the second by Lemma 2 . Continuing in this way, from (1) we get in ... the last term

$$
t_{3^{p}}\left(\cdot,-\delta_{s+1}+\varkappa\left(\gamma_{r}\right) \delta_{r+1}+\cdots+\varkappa\left(\gamma_{q}\right) \delta_{q+1}\right)
$$

for $\gamma_{p}=1$, and this polynomial together with

$$
t_{3^{p}}\left(\cdot,-\delta_{s+1}+\varkappa\left(\gamma_{r}\right) \delta_{r+1}+\cdots+\varkappa\left(\gamma_{q}\right) \delta_{q+1}+\delta_{p+1}\right)
$$

for $\gamma_{p}=2$. The last polynomial has the form $t_{3^{p}}(\cdot, \delta)$ with $\delta>0$, so we can apply Lemma 2 for it. In the case $\gamma_{q}=1$ the previous polynomial is of the same type. If $\gamma_{q}=2$ then we combine the polynomial $t_{3^{p}}\left(\cdot,-\delta_{s+1}+\cdots-\delta_{q+1}\right)$ with $t_{3^{q}}\left(\cdot,-\delta_{s+1}+\cdots+\delta_{q+1}\right)$, which is the second in $q$-th pair. Thus we get the product $t=t_{3^{q}}\left(\cdot, \lambda+\delta_{q+1}\right) \cdot t_{3^{p}}\left(\cdot, \lambda-\delta_{q+1}\right)$ with $\lambda=\sum_{k=q+1}^{s} \varkappa\left(\gamma_{k}\right) \delta_{k+1}$, where $\varkappa\left(\gamma_{s}\right)=-1$. It is easy to check that

$$
-\frac{1}{2} \delta_{q+1}+\frac{1}{2} \delta_{s+1} \leqq \lambda \leqq \frac{1}{2} \delta_{q+1}-\frac{5}{2} \delta_{s+1}
$$

Therefore, $|\lambda|<\frac{1}{2} \delta_{q+1}$ and Lemma 5 can be applied to the polynomial $t$. By that we decompose $\mu_{N}$ into a product of terms every one of which has maximum modulus at 0 , so $\mu_{N}$ also has this property.

\section{On the growth of $\Lambda_{N}$}

Values of the polynomial $t_{n}(\cdot, \delta)$ can be expressed in terms of the product

$$
\pi_{n}(b):=\sin \left(\frac{\pi}{4 n}+b\right) \sin \left(\frac{3 \pi}{4 n}+b\right) \cdots \sin \left(\frac{2 n-1}{4 n} \pi+b\right) .
$$

For example, as is easy to see,

$$
t_{n}(0, \delta)=2^{n} \pi_{n}^{2}(\delta / 2), \quad t_{n}(\pi, \delta)=(-2)^{n} \pi_{n}^{2}(-\delta / 2)
$$

For some values of the parameter $b$, the exact magnitude of $\pi_{n}(b)$ can be found.

LEMma 7. For any $n \in \mathbb{N}$ we have $\pi_{n}(0)=2^{-n+\frac{1}{2}}, \pi_{n}\left(\frac{\pi}{4 n}\right)=2^{-n+1} \sqrt{n}$. 
Proof. Let us show first that for any $m \in \mathbb{N}$

$$
2^{m} \prod_{k=1}^{m} \cos \frac{k \pi}{2 m+1}=1
$$

The Chebyshev polynomial $T_{2 m+1}$ has local extrema at the points $\cos \frac{k \pi}{2 m+1}$ for $k=1, \ldots, 2 m$. Hence,

$$
T_{2 m+1}^{\prime}(x)=2^{2 m}(2 m+1) \prod_{k=1}^{2 m}\left(x-\cos \frac{k \pi}{2 m+1}\right) .
$$

On the other hand,

$$
T_{2 m+1}^{\prime}(x)=(2 m+1) \frac{\sin (2 m+1) \theta}{\sin \theta} \quad \text { for } \quad x=\cos \theta \quad \text { with } \quad 0 \leqq \theta \leqq \pi .
$$

Taking $x=0$ in both expressions yields

$$
T_{2 m+1}^{\prime}(0)=2^{2 m}(2 m+1) \prod_{k=1}^{2 m} \cos \frac{k \pi}{2 m+1}=(2 m+1)(-1)^{m} .
$$

Since

$$
\prod_{k=m+1}^{2 m} \cos \frac{k \pi}{2 m+1}=(-1)^{m} \prod_{k=1}^{m} \cos \frac{k \pi}{2 m+1}
$$

(11) follows.

By means of (11) we can express $\pi_{n}(0)=\prod_{k=1}^{n} \sin \frac{2 k-1}{4 n} \pi$.

Let us first examine the odd value $n=2 m+1$. Here,

$$
\pi_{n}(0)=\prod_{k=1}^{m} \sin \frac{2 k-1}{4 n} \pi \cdot \frac{1}{\sqrt{2}} \cdot \prod_{k=1}^{m} \cos \frac{2 k-1}{4 n} \pi=2^{-m-\frac{1}{2}} \cdot \prod_{k=1}^{m} \sin \frac{2 k-1}{2 n} \pi .
$$

The last product is equal to $\prod_{k=1}^{m} \cos \frac{k \pi}{n}$, which is $2^{-m}$, by (11). Thus, $\pi_{n}(0)=2^{-m-\frac{1}{2}} 2^{-m}=2^{-n+\frac{1}{2}}$.

Similarly, $\pi_{2 m}(0)=2^{-m} \pi_{m}(0)$, and by that, $\pi_{2^{q} k}(0)=2^{-k\left(2^{q}-1\right)} \pi_{k}(0)$ for any $k, q \in \mathbb{N}$. For the general case, we write $n$ in the form $n=2^{q}(2 m+1)$ with $m, q \in \mathbb{N}_{0}$. Combining the previous representations we get

$$
\pi_{n}(0)=2^{-(2 m+1)\left(2^{q}-1\right)} \cdot 2^{-2 m-\frac{1}{2}}=2^{-n+\frac{1}{2}} .
$$


To express $\pi_{n}:=\pi_{n}\left(\frac{\pi}{4 n}\right)=\prod_{k=1}^{n-1} \sin \frac{k \pi}{2 n}$ we apply similar arguments. Instead of (11) we use

$$
2^{m} \prod_{k=1}^{m} \cos \frac{2 k-1}{4 m+2} \pi=\sqrt{2 m+1} \quad \text { for } \quad m \in \mathbb{N} .
$$

Indeed, $T_{2 m+1}(x)=2^{2 m} x \prod_{k=1}^{m}\left(x^{2}-\cos ^{2} \frac{2 k-1}{4 m+2} \pi\right)$. Thus,

$$
T_{2 m+1}^{\prime}(0)=\lim _{x \rightarrow 0} \frac{T_{2 m+1}(x)}{x}=2^{2 m}(-1)^{m} \prod_{k=1}^{m} \cos ^{2} \frac{2 k-1}{4 m+2} \pi .
$$

But as above, $T_{2 m+1}^{\prime}(0)=(-1)^{m}(2 m+1)$. Comparing both forms of $T_{2 m+1}^{\prime}(0)$ we obtain (12).

Arguing as above, we see that $\pi_{2 m}=2^{-m+\frac{1}{2}} \pi_{m}$ and $\pi_{2^{q} k}=2^{\varkappa} \pi_{k}$ with $\varkappa=\left(-2^{q}+1\right) k+q / 2$. Also,

$$
\pi_{2 m+1}=2^{-m} \prod_{k=1}^{m} \sin \frac{k \pi}{2 m+1}=2^{-m} \prod_{k=1}^{m} \cos \frac{2 k-1}{4 m+2} \pi=4^{-m} \sqrt{2 m+1}
$$

by (12). Thus for any $n=2^{q}(2 m+1)$ we have

$$
\pi_{n}=2^{(2 m+1)\left(-2^{q}+1\right)+q / 2} \cdot 4^{-m} \sqrt{2 m+1}=2^{-n+1} \sqrt{n},
$$

which is the desired conclusion.

COROLlary 1. If $0 \leqq \delta \leqq \frac{\pi}{2 n}$ then $2^{1-n} \leqq \max _{0 \leqq \theta \leqq \pi}\left|t_{n}(\theta, \delta)\right| \leqq 2^{2-n} n$. If $-\frac{\pi}{2 n}+\varepsilon \leqq \sigma \leqq 0$ for some $0<\varepsilon<\frac{\pi}{2 n}$ then $\left|t_{n}(\pi, \sigma)\right| / t_{n}(0, \sigma) \leqq(\pi / \varepsilon)^{2}$.

ProOF. Indeed, if $0 \leqq \delta \leqq \frac{\pi}{2 n}$ then by Lemma 4 we have

$$
\max _{0 \leqq \theta \leqq \pi}\left|t_{n}(\theta, \delta)\right|=t_{n}(0, \delta)=2^{n} \pi_{n}^{2}(\delta / 2) \leqq 2^{n} \pi_{n}^{2}\left(\frac{\pi}{4 n}\right)=2^{2-n} n .
$$

Also, $t_{n}(0, \delta) \geqq \tilde{T}_{n}(0)=2^{1-n}$.

To estimate the other term, we note that

$$
\left|t_{n}(\pi, \sigma)\right| \leqq 2^{n} \pi_{n}^{2}\left(\frac{\pi}{4 n}\right)=2^{2-n} n
$$

On the other hand,

$$
\pi_{n}(\sigma / 2) \geqq \sin \varepsilon / 2 \cdot \prod_{k=1}^{n-1} \sin \left(\frac{k \pi}{2 n}+\frac{\varepsilon}{2}\right) \geqq \frac{\varepsilon}{\pi} \pi_{n}\left(\frac{\pi}{4 n}\right),
$$

so $t_{n}(0, \sigma) \geqq 2^{2-n} n\left(\frac{\varepsilon}{\pi}\right)^{2}$. 
Our next objective is to analyze the remaining polynomial

$$
\rho_{N}=\prod_{k=N+1}^{3^{s+1}}\left[\cos (\cdot)-\cos \psi_{k}\right] .
$$

This polynomial can be handled in the same way as $\mu_{N}$ in Lemma 6 .

Lemma 8. Let $3^{s}<N<3^{s+1}$ for some $s \in \mathbb{N}_{0}$, $0 \leqq \theta \leqq \pi$. Then $\left|\rho_{N}(\theta)\right|$ $\leqq 18^{2} \cdot 3^{s^{2}+5 s} \rho_{N}(0)$.

ProOf. Let $M=3^{s+1}-N$, so $\operatorname{deg} \rho_{N}=M$ and $1 \leqq M \leqq 2 \cdot 3^{s}-1$. We use the ternary decomposition

$M=\gamma_{s} \cdot 3^{s}+\gamma_{r} \cdot 3^{r}+\gamma_{q} \cdot 3^{q}+\cdots+\gamma_{p} \cdot 3^{p} \quad$ with $\quad s>r>q>\cdots>p \geqq 0$.

Here $\gamma_{s} \in\{0,1\}$ and $\gamma_{r}, \ldots, \gamma_{p} \in\{1,2\}$. In order to decompose $\rho_{N}$ into a product of polynomials of type $t_{3^{k}}$, we sort out all angles $\left(\psi_{k}\right)_{k=N+1}^{3^{s+1}}$ in the inverse order, that is from $\psi_{3^{s+1}}$ to $\psi_{N+1}$.

Suppose first $\gamma_{s}=1$. Then the angles $\left(\psi_{2 \cdot 3^{s}+k}\right)_{k=1}^{3^{s}}$ are zeros of the polynomial $t_{3^{s}}\left(\cdot,-\delta_{s+1}\right)$. The next $3^{r}$ angles have indices

$$
\left(3^{s}+2 \cdot 3^{s-1}+\cdots+2 \cdot 3^{r}+k\right)_{k=1}^{3^{r}}
$$

and correspond to $t_{3^{r}}\left(\cdot, \delta_{s+1}-\delta_{s}-\cdots-\delta_{r+1}\right)$.

If $\gamma_{s}=0$ then the polynomial $t_{3^{r}}\left(\cdot,-\delta_{s+1}-\delta_{s}-\cdots-\delta_{r+1}\right)$ has zeros at the points $\psi_{j}$ with indices $j=2 \cdot 3^{s}+2 \cdot 3^{s-1}+\cdots+2 \cdot 3^{r}+k$ where $k=1, \ldots, 3^{r}$. Since $\gamma_{s-1}=\cdots=\gamma_{r+1}=0$, so $\varkappa\left(2-\gamma_{k}\right)=-1$ for $r+1 \leqq k \leqq s-1$, the first terms in the decomposition of $\rho_{N}$ are

$$
t_{3^{s}}^{\gamma_{s}}\left(\cdot,-\delta_{s+1}\right) \cdot t_{3^{r}}\left(\cdot, \varkappa\left(2-\gamma_{s}\right) \delta_{s+1}+\cdots+\varkappa\left(2-\gamma_{r+1}\right) \delta_{r+2}-\delta_{r+1}\right) .
$$

If $\gamma_{r}=2$ then the next indices $\left(\left(2-\gamma_{s}\right) 3^{s}+2 \cdot 3^{s-1}+\cdots+3^{r}+k\right)_{k=1}^{3^{r}}$ give

$$
t_{3^{r}}\left(\cdot, \varkappa\left(2-\gamma_{s}\right) \delta_{s+1}+\cdots+\varkappa\left(2-\gamma_{r+1}\right) \delta_{r+2}+\delta_{r+1}\right) .
$$

Continuing in this way, we get

$$
\begin{aligned}
& \rho_{N}= t_{3^{s}}^{\gamma_{s}}\left(\cdot,-\delta_{s+1}\right) \cdot t_{3^{r}}\left(\cdot, \varkappa\left(2-\gamma_{s}\right) \delta_{s+1}+\cdots+\varkappa\left(2-\gamma_{r+1}\right) \delta_{r+2}-\delta_{r+1}\right) \\
& \cdot t_{3^{r}}^{\gamma_{r}-1}\left(\cdot, \varkappa\left(2-\gamma_{s}\right) \delta_{s+1}+\cdots+\varkappa\left(2-\gamma_{r+1}\right) \delta_{r+2}+\delta_{r+1}\right) \cdots \\
& \cdot t_{3^{p}}\left(\cdot, \varkappa\left(2-\gamma_{s}\right) \delta_{s+1}+\cdots+\varkappa\left(2-\gamma_{p+1}\right) \delta_{p+2}-\delta_{p+1}\right) \cdot t_{3^{p}}^{\gamma_{p}-1}\left(\cdot, \cdots+\delta_{p+1}\right) .
\end{aligned}
$$


For every term of type $t_{3^{k}}^{\gamma_{k}-1}(\cdot, \delta)$ we have $\delta>0$. Therefore, $\left|t_{3^{k}}^{\gamma_{k}-1}(\theta, \delta)\right|$ $\leqq t_{3^{k}}^{\gamma_{k}-1}(0, \delta)$ for any $0 \leqq \theta \leqq \pi$, by Lemma 4 , and we can neglect these terms while estimating $\left|\rho_{N}(\theta)\right| / \rho_{N}(0)$ from above. All other terms (with $\delta<0$ ) attain the maximal modulus value at $\theta=\pi$, by Lemma 4 . It follows that

$$
\frac{\left|\rho_{N}(\theta)\right|}{\rho_{N}(0)} \leqq \frac{\left|t_{3^{s}}^{\gamma_{s}}\left(\pi,-\delta_{s+1}\right)\right|}{t_{3^{s}}^{\gamma_{s}}\left(0,-\delta_{s+1}\right)} \cdot \prod_{k=p, \ldots, q, r} \frac{\left|t_{3^{k}}\left(\pi, \sigma_{k}\right)\right|}{t_{3^{k}}\left(0, \sigma_{k}\right)}
$$

where $\sigma_{k}=\varkappa\left(2-\gamma_{s}\right) \delta_{s+1}+\cdots+\varkappa\left(2-\gamma_{k+1}\right) \delta_{k+2}-\delta_{k+1}$.

Suppose $\gamma_{s}=0$. Then the first term on the right in (13) is absent. Let us estimate all $\sigma_{k}$ from above. At first, $\sigma_{r}=-\delta_{s+1}-\delta_{s}-\cdots-\delta_{r+1}$ $=-\frac{1}{2} \delta_{r}+\frac{1}{2} \delta_{s+1}$. From Corollary 1 we conclude that $\left|t_{3^{r}}\left(\pi, \sigma_{r}\right)\right| / t_{3^{r}}\left(0, \sigma_{r}\right)$ $\leqq\left(2 \pi / \delta_{s+1}\right)^{2}=4 \cdot 3^{2(s+1)}$. Secondly,

$$
\sigma_{q}=-\delta_{s+1}-\delta_{s}-\cdots-\delta_{r+2}+\varkappa\left(2-\gamma_{r}\right) \delta_{r+1}-\delta_{r}-\cdots-\delta_{q+2}-\delta_{q+1} .
$$

But here $\gamma_{r} \neq 0$, so $\varkappa\left(2-\gamma_{r}\right) \geqq 0$. Therefore,

$$
\sigma_{q} \geqq-\frac{1}{2} \delta_{q}+\frac{1}{2} \delta_{r}-\frac{1}{2} \delta_{r+1}+\frac{1}{2} \delta_{s+1}>-\frac{1}{2} \delta_{q}+\delta_{r+1} .
$$

In the worst case, when $r=s-1$, we get

$$
\sigma_{q}>-\frac{1}{2} \delta_{q}+\delta_{s} \quad \text { and } \quad\left|t_{3^{q}}\left(\pi, \sigma_{q}\right)\right| / t_{3^{q}}\left(0, \sigma_{q}\right) \leqq 3^{2 s}
$$

In general,

$$
\begin{gathered}
\sigma_{k}=-\delta_{s+1}-\delta_{s}-\cdots-\delta_{r+2}+\varkappa\left(2-\gamma_{r}\right) \delta_{r+1}-\delta_{r}-\cdots-\delta_{m+2} \\
+\varkappa\left(2-\gamma_{m}\right) \delta_{m+1}-\delta_{m}-\cdots-\delta_{k+2}-\delta_{k+1}
\end{gathered}
$$

where $m=\min \left\{j>k: \gamma_{j} \neq 0\right\}$. Thus, $\sigma_{k}>-\frac{1}{2} \delta_{k}+\delta_{m+1}$ and the product on the right in (13) can be estimated from above by

$$
4 \cdot 3^{2(s+1)} 3^{2 s} \cdots 3^{2}=4 \cdot 3^{(s+2)(s+1)}=36 \cdot 3^{s^{2}+3 s} .
$$

For the case $\gamma_{s}=1$ we apply similar arguments. Here,

$$
-\delta_{s+1}=-\frac{1}{2} \delta_{s}+\frac{1}{2} \delta_{s+1}, \quad \text { so } \quad\left|t_{3^{s}}\left(\pi,-\delta_{s+1}\right)\right| / t_{3^{s}}\left(0,-\delta_{s+1}\right) \leqq 4 \cdot 3^{2(s+1)} .
$$


Also,

$$
\sigma_{r}=\delta_{s+1}-\delta_{s}-\cdots-\delta_{r+1}=-\frac{1}{2} \delta_{r}+\frac{5}{6} \delta_{s}>-\frac{1}{2} \delta_{r}+\delta_{s+1} .
$$

For any other value of $k$ the lower bound of $\sigma_{k}$ is the same as for the case $\gamma_{s}=0$. Therefore,

$\left|\rho_{N}(\theta)\right| / \rho_{N}(0) \leqq 4 \cdot 3^{2(s+1)} \cdot 3^{2(s+1+s+\cdots+1)}=4 \cdot 3^{(s+1)(s+4)}=18^{2} \cdot 3^{s^{2}+5 s}$.

We are now able to estimate the fundamental Lagrange polynomials corresponding to the given sequence.

Lemma 9. For any $N \in \mathbb{N}, 1 \leqq k \leqq N+1$, and $|x| \leqq 1$, we have $\left|l_{k, N}(x)\right|$ $\leqq 4 \pi N^{2}\left|l_{k, N}(1)\right|$

Proof. If $N=1$ then for both polynomials $l_{1,1}(x)=-\frac{2}{\sqrt{3}} x$ and $l_{2,1}(x)$ $=\frac{2 x+\sqrt{3}}{\sqrt{3}}$ we have trivially $\left|l_{k, 1}(x)\right| \leqq\left|l_{k, 1}(1)\right|$.

If $N=3^{s}-1$ for some $s \in \mathbb{N}$, then $l_{k, N}(x)=\frac{T_{3} s(x)}{\left(x-x_{k}\right) T_{3^{s}}^{\prime}\left(x_{k}\right)}$ and $\left|l_{k, N}(x)\right|$ $\leqq 4 / \pi$ (see e.g. [5], p. 58). On the other hand,

$$
\left|l_{k, N}(1)\right|=\left[\left(1-x_{k}\right) T_{3^{s}}^{\prime}\left(x_{k}\right)\right]^{-1} \geqq 3^{-2 s},
$$

by the Markov inequality (see e.g. [5], p. 123), which gives the result for this case. Here,

Suppose $3^{s} \leqq N \leqq 3^{s+1}-2$ for some $s \in \mathbb{N}$. Fix $k \leqq N+1$ and $0 \leqq \theta \leqq \pi$.

$$
l_{k, N}(\cos \theta)=\frac{\mu_{N+1}(\theta)}{\left(\cos \theta-\cos \psi_{k}\right) \omega_{N+1}^{\prime}\left(x_{k}\right)}
$$

and the desired inequality has the form

$$
\frac{1-\cos \theta}{\left|\cos \theta-\cos \psi_{k}\right|} \cdot \frac{\left|\mu_{N+1}(\theta)\right|}{\mu_{N+1}(0)} \leqq 4 \pi N^{2}
$$

The angle $\psi_{k}$ is equal to $\left(i-\frac{1}{2}\right) \delta_{s+1}$ with some $1<i \leqq 3^{s+1}$. The value $i=1$ is excluded since in this case we have $\psi_{k}=\frac{1}{2} \delta_{s+1}$, that is $k=3^{s+1}$, but $k \leqq N+1 \leqq 3^{s+1}-1$. Thus, $\frac{3}{2} \delta_{s+1} \leqq \psi_{k} \leqq \pi-\frac{1}{2} \delta_{s+1}$.

Let us first consider the case $\left|\theta-\psi_{k}\right|>\frac{1}{2} \delta_{s+1}$. Here, $\frac{3}{2} \delta_{s+1} \leqq \theta+\psi_{k} \leqq$ $2 \pi-\frac{3}{2} \delta_{s+1}$. Indeed, if $\psi_{k}=\pi-\frac{1}{2} \delta_{s+1}$ then $\theta<\pi-\delta_{s+1}$. Otherwise, $\psi_{k} \leqq$ $\pi-\frac{3}{2} \delta_{s+1}$ with any $\theta \leqq \pi$. 
Therefore,

$$
\begin{aligned}
\left|\cos \theta-\cos \psi_{k}\right| & =2 \sin \frac{1}{2}\left|\theta-\psi_{k}\right| \cdot \sin \frac{1}{2}\left(\theta+\psi_{k}\right)>2 \sin \frac{1}{4} \delta_{s+1} \cdot \sin \frac{3}{4} \delta_{s+1} \\
& >2 \frac{4}{\pi^{2}} \frac{1}{4} \delta_{s+1} \frac{3}{4} \delta_{s+1}=\frac{1}{6} \cdot 3^{-2 s} \geqq \frac{1}{6} \cdot N^{-2} .
\end{aligned}
$$

This inequality together with Lemma 6 imply (14).

For the case $\left|\theta-\psi_{k}\right| \leqq \frac{1}{2} \delta_{s+1}$ we use the representation

$$
\mu_{N+1}(\theta)=\mu_{N+1}\left(\psi_{k}\right)+\mu_{N+1}^{\prime}(\xi) \cdot\left(\theta-\psi_{k}\right)
$$

and the Bernstein inequality (see e.g. [5], p. 118):

$$
\left|\mu_{N+1}^{\prime}(\xi)\right| \leqq(N+1) \cdot \max _{0 \leqq \theta \leqq \pi}\left|\mu_{N+1}(\theta)\right|
$$

Since $\mu_{N+1}\left(\psi_{k}\right)=0$, we get by Lemma 6 ,

$$
\mu_{N+1}(\theta) \leqq(N+1) \cdot\left|\theta-\psi_{k}\right| \cdot \mu_{N+1}(0) .
$$

To prove (14) it is enough to show

$$
(N+1) \cdot\left|\theta-\psi_{k}\right| \leqq 4 \pi N^{2}\left|\cos \theta-\cos \psi_{k}\right| .
$$

Here, $\frac{3}{2} \delta_{s+1} \leqq \theta+\psi_{k} \leqq 2 \pi-\frac{1}{2} \delta_{s+1}$. Therefore, $\sin \frac{1}{2}\left(\theta+\psi_{k}\right) \geqq \sin \frac{1}{4} \delta_{s+1}$ $>\frac{1}{6 N}$. On the other hand, $\sin \frac{1}{2}\left|\theta-\psi_{k}\right|>\frac{1}{\pi}\left|\theta-\psi_{k}\right|$. Hence, $\left|\cos \theta-\cos \psi_{k}\right|>$ $\left|\theta-\psi_{k}\right|(3 \pi N)^{-1}$, which gives (15), since $N+1 \leqq \frac{4}{3} N$ for $N \geqq 3$.

THEOREM 1. For the Lebesgue constants $\Lambda_{N}(X)$ corresponding to the Newton interpolation at the points $X=\left(x_{n}\right)_{n=1}^{\infty}$ we have

$$
\Lambda_{N}(X) \leqq C \cdot N^{8} \cdot N^{\log N / \log 3} \quad \text { for any } \quad N \geqq 1 .
$$

Here $C=2 \cdot 72^{2}$.

Proof. Let $N_{s}=3^{s}-1$. If $N=N_{s}$ then $\left(x_{k}\right)_{k=1}^{N+1}$ are exactly the zeros of $T_{3^{s}}$ and (see e.g. [5], p. 19) $\Lambda_{N}(X) \leqq \frac{2}{\pi} \log N+1$. Therefore we can make the assumption: $3^{s} \leqq N \leqq 3^{s+1}-2$ for some $s \in \mathbb{N}_{0}$. For such $N$ we have

$$
\left|l_{k, N}(1)\right|=\left|l_{k, N_{s+1}}(1)\right| \cdot \frac{\left|\rho_{N+1}\left(\psi_{k}\right)\right|}{\rho_{N+1}(0)} .
$$


For the Lagrange fundamental polynomials at Chebyshev's points we have $\left|l_{k, N_{s+1}}(1)\right| \leqq 4 / \pi$ (see for instance [5], p. 58).

Lemma 9 and Lemma 8 now show that for any $|x| \leqq 1$ we have

$$
\left|l_{k, N}(x)\right| \leqq 16 \cdot 18^{2} \cdot N^{2} \cdot 3^{s^{2}+5 s} \leqq 72^{2} \cdot N^{7} \cdot 3^{s^{2}} .
$$

This gives the desired result, since $\Lambda_{N}(X)$ is a sum of $N+1$ terms with bound presented above and $N+1 \leqq 2 N$.

ExAmple. Let us estimate from below the value $\left|l_{j, N}(1)\right|$ for $N=$ $\frac{1}{2}\left(3^{s+1}-1\right)$ and $j=\frac{1}{2}\left(3^{s}+1\right)$. Here, $\psi_{j}=\max _{1 \leqq k \leqq N} \psi_{k}=\pi-\frac{1}{2} \delta_{s}$. Therefore, $x_{j}=-\cos \frac{1}{2} \delta_{s}$. Since $N=3^{s}+3^{s-1}+\cdots+3+1$, the polynomial $\mu_{N}$ has the decomposition $\mu_{N}=\tilde{T}_{3^{s}} \prod_{q=0}^{s-1} t_{3 q}\left(\cdot, \delta_{q+2}+\cdots+\delta_{s+1}\right)$. Hence,

$$
l_{j, N}(1)=l_{j, N_{s}}(1) \cdot \prod_{q=0}^{s-1} \frac{t_{3^{q}}\left(0, \sigma_{q}\right)}{t_{3^{q}}\left(\psi_{j}, \sigma_{q}\right)}
$$

with $\sigma_{q}=\frac{1}{2}\left(\delta_{q+1}-\delta_{s+1}\right)$.

Here the value of $l_{j, N_{s}}(1)$ is rather small. Indeed,

$$
l_{j, N_{s}}(1)=\frac{T_{3^{s}}(1)}{T_{3^{s}}^{\prime}\left(x_{j}\right)\left(1-x_{j}\right)}=3^{-s} \cdot \tan \frac{1}{4} \delta_{s}>\frac{\pi}{4 \cdot 9^{s}} .
$$

But because of the positivity of all $\sigma_{q}$, the product on the right side of (16) has a growth of order at least $3^{s^{2} / 3}$, as we will see now. Since all terms in the product are larger than 1 , we can exclude the value $q=0$. For $1 \leqq q \leqq s-1$, let $K=\frac{1}{2}\left(3^{q}-1\right)$. It is easily seen that $t_{3^{q}}\left(0, \sigma_{q}\right) /\left|t_{3^{q}}\left(\psi_{j}, \sigma_{q}\right)\right|>A_{q}^{2}$, where

$$
\begin{gathered}
A_{q}=\prod_{k=1}^{3^{q}} \tan \left(\frac{3 k-1}{6} \delta_{q}-\frac{1}{4} \delta_{s+1}\right)=\cot \left(\frac{3 K+1}{6} \delta_{q}+\frac{1}{4} \delta_{s+1}\right) \\
\cdot \prod_{k=1}^{K} \tan \left(\frac{3 k-1}{6} \delta_{q}-\frac{1}{4} \delta_{s+1}\right) / \tan \left(\frac{3 k-2}{6} \delta_{q}+\frac{1}{4} \delta_{s+1}\right) .
\end{gathered}
$$

The first cotangent is larger than 1 since its argument does not exceed $\pi / 4$. Also we can neglect the term in the product corresponding the value $k=1$. For other terms we use the formula

$$
\frac{\tan v}{\tan u}=1+\tan (v-u) \cdot(\cot u+\tan v) .
$$


From here,

$$
\log A_{q}>\sum_{k=2}^{K} \log \left(1+a_{k}\right)>\sum_{k=2}^{K} a_{k}-\frac{1}{2} \sum_{k=2}^{K} a_{k}^{2}
$$

where

$$
\begin{gathered}
a_{k}=\tan \left(\frac{1}{6} \delta_{q}-\frac{1}{2} \delta_{s+1}\right) \cdot\left[\cot \left(\frac{3 k-2}{6} \delta_{q}+\frac{1}{4} \delta_{s+1}\right)\right. \\
\left.+\tan \left(\frac{3 k-1}{6} \delta_{q}-\frac{1}{4} \delta_{s+1}\right)\right]
\end{gathered}
$$

The expression in square brackets can be written as

$$
\cot (b-\varepsilon)+\tan (b+\varepsilon)=\frac{2 \cos 2 \varepsilon}{\sin 2 b-\sin 2 \varepsilon}
$$

with $b=\frac{1}{2}\left(k-\frac{1}{2}\right) \delta_{q}, \quad \varepsilon=\frac{1}{12} \delta_{q}-\frac{1}{4} \delta_{s+1}$. Here, $b>9 \varepsilon$, so $[\cdots]<\frac{8}{(2 k-1) \delta_{q}}$. Therefore, $\sum_{k=2}^{K} a_{k}^{2}<\frac{1}{2}$, as is easy to check.

In order to estimate $a_{k}$ from below, we use the bound $\cot t>\frac{1}{t}-\frac{t}{2}$, which is valid for $0<t<\pi$. It follows that

$$
a_{k}>\left(\frac{1}{6} \delta_{q}-\frac{1}{2} \delta_{s+1}\right) \cdot \cot \left(\frac{1}{2} k \delta_{q}\right)>\left(\frac{1}{6} \delta_{q}-\frac{1}{2} \delta_{s+1}\right) \cdot\left(\frac{2}{k \delta_{q}}-\frac{k \delta_{q}}{4}\right) .
$$

Straightforward estimation gives $\sum_{k=2}^{K} a_{k}>\varkappa \cdot \log K-0.193$, where $\varkappa=3^{-1}$ $-3^{q-s-1}$. Therefore, $A_{q}>3^{q \cdot \varkappa} \cdot e^{-0.43}$ and

$$
\prod_{q=1}^{s-1} t_{3^{q}}\left(0, \sigma_{q}\right) /\left|t_{3^{q}}\left(\psi_{j}, \sigma_{q}\right)\right|>e^{-0.86 \cdot s} \cdot 3^{\varkappa 1}
$$

with

$$
\varkappa_{1}=\frac{1}{3} s(s-1)-2 \cdot 3^{-s-1} \sum_{q=1}^{s-1} q 3^{q} .
$$

Since the last sum is equal to $\frac{1}{4} 3^{s}(2 s-3)+\frac{3}{4}$, we have $\varkappa_{1}>\frac{1}{3} s^{2}-\frac{2}{3} s$. Eventually,

$$
\left|l_{j, N}(1)\right|>\frac{\pi}{4}\left(e^{0.86} \cdot 3^{8 / 3}\right)^{-s} \cdot 3^{s^{2} / 3}>3^{s^{2} / 3-4 s} .
$$

The latter exceeds $\exp \left(\frac{\log ^{2} N_{s}}{\log 27}-5 \log N_{s}\right)$, as is easy to check. 
Question. One can suggest that more symmetric infill of the levels $Z_{s}$ will decrease the size of the Lebesgue constants corresponding to a new sequence. In this connection we can reformulate the question from [2]: Is it possible to reach a polynomial growth of the sequence $\left(\Lambda_{N}(X)\right)_{N=0}^{\infty}$ for some another rearrangement of points from the sequence $\left(x_{k}\right)_{k=1}^{\infty}$ ?

\section{References}

[1] L. Brutman, Lebesgue functions for polynomial interpolation - a survey, Ann. Numer. Math., 4 (1997), 111-127.

[2] A. Goncharov, What is the size of the Lebesgue constant for Newton interpolation?, in: Constructive Theory of Functions (Varna, 2005), edited by B. D. Bojanov, p. 144.

[3] E. Levin and B. Shekhtman, Two problems on interpolation, Constr. Approx., 11 (1995), 513-515.

[4] Paul G. Nevai, Orthogonal Polynomials, Memoirs of AMS, Vol.18, 123 (Providence, 1979).

[5] T. J. Rivlin, The Chebyshev Polynomials, second edition, Pure and Applied Mathematics (New York, 1990).

[6] E. B. Saff and V. Totik, Logarithmic Potentials with External Fields, Springer-Verlag (1997).

[7] G. Szegö, Orthogonal Polynomials, fourth edition, AMS Coll. Publ., Vol. 23 (Providence, 1975).

[8] R. Taylor and V. Totik, Lebesgue constants for Leja points, IMA Journal of Num. Anal. (to appear). 